\title{
Optical response of fluorescent molecules studied by synthetic femtosecond laser pulses
}

\author{
A. Konar ${ }^{1}$, J.-D. Shah ${ }^{1}$, V.-V. Lozovoy ${ }^{1}$, and M. Dantus ${ }^{1,2}$ \\ ${ }^{1}$ Department of Chemistry, Michigan State University, East Lansing, MI 48824, USA \\ ${ }^{2}$ Department of Physics and Astronomy, Michigan State University, East Lansing, MI 48824, USA
}

\begin{abstract}
The optical response of the fluorescent molecule IR144 in solution is probed by pairs of collinear pulses with intensity just above the linear dependence using two different pulse shaping methods. The first approach mimics a Michelson interferometer while the second approach, known as multiple independent comb shaping (MICS), eliminates spectral interference. The comparison of interfering and non-interfering pulses reveals that experimental information can be lost at early delay times because of linear interference between the pulses.
\end{abstract}

\section{Introduction}

The optical response of molecules in solution to a pulse of light has been the subject of numerous studies $[1,2]$ that have evolved with technology from the microsecond to the femtosecond timescale. When femtosecond laser pulses are temporally overlapped, it is difficult to isolate the molecular response from pulse interference effects. This general difficulty has often been mitigated by ignoring the early time dynamics, while the pulses are overlapped, or by subtracting the early response as generated by the pure solvent. For example, when two collinear identical pulses, such as those generated by a Michelson interferometer, are scanned in time, they optically interfere, causing different amplitude modulation of the laser spectrum at different delay times. This interference may overwhelm the molecular dynamics that one wishes to measure. This simple fact has inspired experimental setups capable of measuring the early optical response of molecules with nearly identical pulses that do not interfere. One such setup, multiple independent comb shaping (MICS) [3], introduced by Pestov et al, is used here to study a model system, IR-144, chosen due to its wellknown fluorescent properties in solution. This article compares the two experimental approaches (Michelson and MICS) and illustrates how MICS is able to obtain the expected molecular behavior without imposing spectral interference.

\section{Experimental}

The output from a $1 \mathrm{kHz}$ amplifier centered at $800 \mathrm{~nm}$ is $\sim 700 \mu \mathrm{J}$ and was attenuated before entering a phase-amplitude pulse shaper as shown in Figure 1a. The dispersed spectrum covered 600 SLM pixels with a resolution of $0.32 \mathrm{~nm}$ per pixel. High-order phase distortions introduced by the optics in the laser system as well as by the setup are compensated by the multiphoton intrapulse interference phase scan (MIIPS) [4] software resulting in 35 fs transform limited (TL) pulses at the sample.

This is an Open Access article distributed under the terms of the Creative Commons Attribution License 2.0, which permits unrestricted use, distribution, and reproduction in any medium, provided the original work is properly cited. 


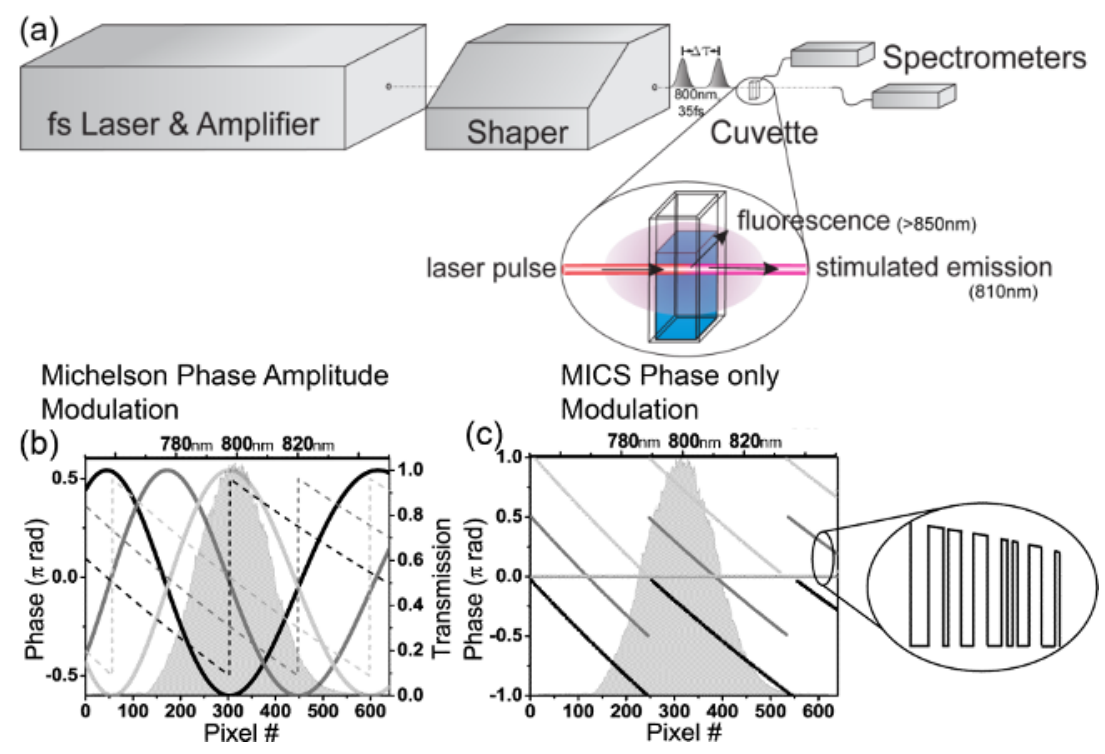

Fig. 1. Experimental setup and two different pulse shaper parameters used to create interfering and noninterfering pairs of pulses. (a) Experimental setup for collection of fluorescence at right angles and stimulated emission from the sample. (b) Phase (solid) and amplitude (dashed) mask corresponding to an interferometric scan with delays of $25.27 \mathrm{fs}$ (black), $25.935 \mathrm{fs}$ (gray) and $26.6 \mathrm{fs}$ (light gray). (c) Phase mask corresponding to MICS scan with the same delay times as in (b)

Examples of the phase and amplitude modulations required for pulses separated by $25.27 \mathrm{fs}$ (out of phase), 25.935 fs (in quadrature) and 26.6 fs (in phase) are shown in Figure 1b. Pairs of noninterfering replica pulses at the same delays were prepared using the MICS approach. Experimentally this is done by decomposing the spectrum into two independent frequency combs that sample the entire bandwidth as shown in Figure 1c. Each comb is then addressed by a linear function with a slope that corresponds to the time delay being introduced. Because each of the two frequency combs has no frequencies in common, when the two pulses are scanned with respect to each other, they experience no spectral interference by design. Both methods for creating pulse replicas were tested first by collecting the fundamental spectrum as a function of time delay between the pulses as shown in Figures $2 \mathrm{a}$ and b. The MICS scan shows little or no optical interference, as desired. Note that the phase-amplitude modulation scan shows the expected interference between the two pulses at the optical frequency $\sim 2.66 \mathrm{fs}$. The MICS scan shows little or no optical interference, as desired. At long delay times, when using interfering pulses, half of the amplitude is lost in the transmission mask. For MICS, half of the intensity of the pulses appears as broad satellites approximately 1 ps away from the central pulses.

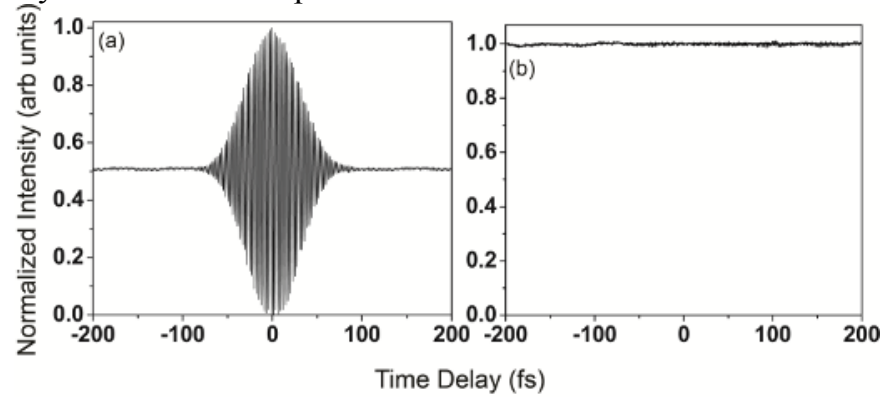

Fig. 2. Interferometric time delay scans for the fundamental signal obtained using (a) a pair of transform limited interfering pulses using phase amplitude modulation and (b) a pair of non-interfering pulses using MICS. 


\section{Results and Discussion}

Fluorescence and stimulated emission scans obtained as a function of delay time were first obtained using a pair of interfering pulses, as shown in the experimental part of Figure $3 \mathrm{c}$ and $3 \mathrm{~d}$ respectively. The peak power density for transform limited (TL) pulses is calculated to be $10^{10} \mathrm{~W} / \mathrm{cm}^{2}$. Interference fringes are observed at the optical frequency and the amplitude is found to be greater when the two pulses interfere constructively than when they are separated at long times. The stimulated emission scan is very similar to the fluorescence scan. Note that both the fluorescence and stimulated emission scans are very similar to the linear autocorrelations shown in Figure 2. Similar scans were obtained as a function of time delay using non-interfering pulse replica based on MICS, as shown in Figure $3 \mathrm{a}$ and $3 \mathrm{~b}$ using the same peak power and dye concentration. A close look at the region near zero delay time shows that the fringes observed for fluorescence and stimulated emission are exactly out of phase. This is expected near saturation, when the observed fluorescence is lower. Another striking feature in the fluorescence is the initial dip in the signal which has been successfully reproduced in the simulation.
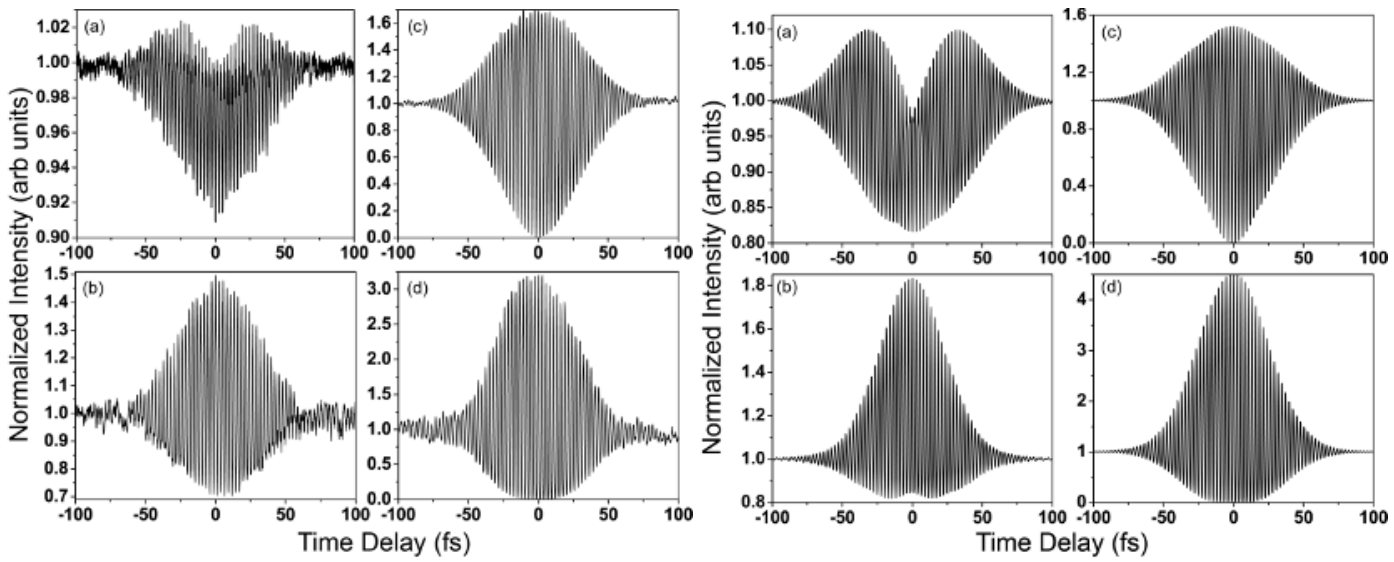

Fig. 3. Experimental (left) and simulated (right) results (a) Integrated fluorescence and (b) stimulated emission signal as a function of time delay of the pulse replicas generated using MICS phase mask. (c) Integrated fluorescence and (d) stimulated emission signal as a function of time delay of the pulse replicas generated using phase and amplitude modulation.

The system is modeled as a two level system and the simulation is the solution of the Liouville equation with dephasing $\left(\mathrm{T}_{2}=100 \mathrm{fs}\right)$ and without relaxation. The laser pulse centered at $800 \mathrm{~nm}$ is detuned from the absorption maxima at $750 \mathrm{~nm}$. Fluorescence is calculated as the population of the excited state at the end of the pulse. Stimulated emission is calculated as the integral of the product between the intensity of the laser and the population of the excited state.

Acknowledgements: We thank DOE SISGR (DE-SC0002325), Dr. Jeff Krause Program Manager for support of this research. We also thank Drs. Dmitry Pestov and Haowen Li of Biophotonic Solutions Inc for generously sharing their pulse shaping expertise.

\section{References}

1. T. H. Joo, Y. W. Jia, J. Y. Yu, M. J. Lang and G. R. Fleming, J. Chem. Phys. 104, 6089 (1996)

2. R. M. Stratt and M. Maroncelli, J. Phys. Chem. 100, 12981 (1996)

3. D. Pestov, V. V. Lozovoy and M. Dantus, Opt Express, 17, 14351 (2009)

4. Y. Coello, V. V. Lozovoy, T. C. Gunaratne, B. Xu, I. Borukhovich, C.Tseng, T.Weinacht, and M. Dantus, J. Opt. Soc. Am. B. 25, A140 (2008) 